\title{
Correction to: Monoallelic KIF1A-related disorders: a multicenter cross sectional study and systematic literature review
}

\author{
Stefania Della Vecchia ${ }^{1}$ - Alessandra Tessa ${ }^{1} \cdot$ Claudia Dosi $^{2} \cdot$ Jacopo Baldacci $^{3} \cdot$ Rosa Pasquariello $^{1}$. \\ Antonella Antenora ${ }^{4}$. Guja Astrea ${ }^{1} \cdot$ Maria Teresa Bassi $^{5} \cdot$ Roberta Battini $^{1,6}$. Carlo Casali ${ }^{7}$. Ettore Cioffi ${ }^{7}$. \\ Greta Conti $^{8}$. Giovanna De Michele ${ }^{4}$. Anna Rita Ferrari ${ }^{1} \cdot$ Alessandro Filla $^{4}$. Chiara Fiorillo ${ }^{9}$. Carlo Fusco ${ }^{10}$. \\ Salvatore Gallone ${ }^{11}$. Chiara Germiniasi ${ }^{12}$ - Renzo Guerrini ${ }^{8}$. Shalom Haggiag ${ }^{13}$. Diego Lopergolo ${ }^{1,14}$. \\ Andrea Martinuzzi ${ }^{15}$. Federico Melani ${ }^{8}$. Andrea Mignarri ${ }^{14}$. Elena Panzeri ${ }^{5}$. Antonella Pini ${ }^{16}$. Anna Maria Pinto ${ }^{17}$. \\ Francesca Pochiero ${ }^{18}$. Guido Primiano ${ }^{19}$. Elena Procopio ${ }^{18}$. Alessandra Renieri ${ }^{17}$. Romina Romaniello ${ }^{20}$. \\ Cristina Sancricca $^{19}$. Serenella Servidei ${ }^{19,21}$. Carlotta Spagnoli ${ }^{10}$. Chiara Ticci ${ }^{1,18}$. Anna Rubegni ${ }^{1}$. \\ Filippo Maria Santorelli ${ }^{1}$
}

Published online: 15 October 2021

(c) The Author(s), under exclusive licence to Springer-Verlag GmbH Germany 2021

\section{Correction to: Journal of Neurology https://doi.org/10.1007/s00415-021-10792-3}

Unfortunately, the given name and family name of the first author was incorrectly tagged in the $\mathrm{xml}$ data, therefore it is

The original article can be found online at https://doi.org/10.1007/ s00415-021-10792-3.

\section{Alessandra Tessa}

aletessa@gmail.com

$\triangle$ Filippo Maria Santorelli filippo3364@gmail.com

1 IRCCS Stella Maris Foundation, Calambrone, via dei Giacinti 2, 56128 Pisa, Italy

2 Child Neurology, Fondazione IRCCS Istituto Neurologico Carlo Besta, 20133 Milan, Italy

3 Kode Solutions, Lungarno Galileo Galilei 1, 56125 Pisa, Italy

4 Department of Neurosciences, Reproductive and Odontostomatological Sciences, Federico II University, 80131 Naples, Italy

5 Laboratory of Molecular Biology, Scientific Institute IRCCS Eugenio Medea, Bosisio Parini, 23842 Lecco, Italy

6 Department of Clinical and Experimental Medicine, Neurological Institute, University of Pisa, 56125 Pisa, Italy

7 Department of Medical and Surgical Sciences and Biotechnologies, Sapienza University of Rome, 40100 Latina, Italy

8 Neurology Unit and Neurogenetics Laboratories, Meyer Children University Hospital, University of Florence, 50139 Florence, Italy

9 Neuromuscular Disorders Unit, IRCCS Istituto Giannina Gaslini, DINOGMI, University of Genoa, Genoa, Italy abbreviated wrongly in Pubmed. The correct given name is Stefania and family name is Della Vecchia.

10 Child Neurology Unit, Pediatric Neurophysiology Laboratory, Department of Pediatrics, Azienda USL-IRCCS Di Reggio Emilia, 42122 Reggio Emilia, Italy

11 Clinical Neurogenetics, Department Neurosciences, Az. Osp. Città della Salute e della Scienza di Torino, 1026 Torino, Italy

12 Neuromuscular Unit, Scientific Institute IRCCS E. Medea, Bosisio Parini, 23842 Lecco, Italy

13 Department of Neurology, Azienda Ospedaliera San Camillo Forlanini, 00152 Rome, Italy

14 Unit of Neurology and Neurometabolic Disorders, Department of Medicine, Surgery and Neurosciences, University of Siena, 53100 Siena, Italy

15 Scientific Institute IRCCS E. Medea, Unità Operativa Conegliano, 31015 Treviso, Italy

16 Neuromuscular Pediatric Unit, IRRCS Istituto delle Scienze Neurologiche di Bologna, 40139 Bologna, Italy

17 Medical Genetics Unit, University of Siena, Azienda Ospedaliera Universitaria Senese, 53100 Siena, Italy

18 Department of Metabolic and Muscular, Meyer Children's University Hospital, 50139 Florence, Italy

19 Neurofisiopathology Unit, Fondazione Policlinico Universitario Agostino Gemelli IRCCS, 00168 Rome, Italy

20 Neuropsychiatry and Neurorehabilitation Unit, Scientific Institute, IRCCS Eugenio Medea, Bosisio Parini, 23842 Lecco, Italy

21 Dipartimento Universitario di Neuroscienze, Università Cattolica del Sacro Cuore, 00168 Rome, Italy 